\title{
DEMONSTRATION OF SHARED ANTIGENS IN THE GENUS CLOSTRIDIUM BY AN ENZYME-LINKED IMMUNOSORBENT ASSAY
}

\author{
I. R. Poxton and Marie D. Byrne
}

\section{Bacteriology Department, University of Edinburgh Medical School, Teviot Place, Edinburgh EH8 $9 A G$}

\begin{abstract}
Summary. Antigens prepared from several strains of each of 10 Clostridium species were used in an indirect enzyme-linked immunosorbent assay with antisera raised against whole cells of a representative strain from each of the 10 species killed by ultra-violet irradiation. With the exception of $C$. cadaveris, the antisera gave similar results with antigens prepared from all strains of the homologous species. Antigens prepared from 13 other clostridial species were then investigated in an ELISA system with the 10 representative antisera. The results showed many cross-reactions, particularly in the $C$. perfringens group, the $C$. difficile/sordelli group and the $C$. botulinum/novyi/sporogenes group.
\end{abstract}

\section{INTRODUCTION}

Bacteria in the genus Clostridium are subdivided into species according to a range of morphological and biochemical criteria that include shape, position of spore, the ability to digest proteins and ferment various sugars and the production of a range of extracellular enzymes and toxins. Several identification schemes are available, e.g. those of Holdeman, Cato and Moore (1977) and Willis (1969), but in practice a number of isolates prove difficult to identify. Many species are closely related, and morphological and biochemical variations occur within strains of the same species and even within a subculture of the same strain. Toxigenic and non-toxigenic variants of the same species occur and some species can be distinguished only by differences in the toxins they produce (Cruickshank et al., 1975).

In human and verterinary medicine, immunological methods have been exploited to detect and identify clostridia. Immunofluorescent antibody (IFA) kits are commercially available for $C$. botulinum types A, C and E, C. septicum, C. chauvoei, $C$. novyi and $C$. tetani (Wellcome Diagnostics, Dartford, England), for the detection of $C$. botulinum types $\mathrm{A}, \mathrm{B}$ and $\mathrm{F}$ in suspected infant botulism (Hatheway and McCroskey, 1981 ) and for $C$. difficile in cases of antibiotic associated pseudomembranous colitis (Hubert, Ionesco and Sebald, 1981). Several studies of antigenic relationships in the genus Clostridium have been based on agglutination tests (Mandia, 1955) or empirical immunofluorescence reactions (Walker and Batty, 1964; Batty and Walker, 1965; Lynt, Solomon and Kautter, 1971) with a range of antisera raised against heat-killed or 
formalin-killed organisms. More recently, we presented evidence for antigenic relationships between $C$. difficile and $C$. bifermentans and $C$. sordelli (Poxton and Byrne, 1981a) based on surface antigens extracted by EDTA. These were demonstrated by an enzyme-linked immunosorbent assay (ELISA) and crossed immunoelectrophoresis and we were able to provide guidelines for the detection of $C$. difficile by immunotechniques (Poxton and Byrne, 1981 b).

The work reported here extends these studies to the majority of medically important species of Clostridium.

\section{MATERIALS AND METHODS}

Bacterial strains. The following strains were obtained from the National Collection of Type Cultures (NCTC), Central Public Health Laboratory, Colindale Avenue, London NW9 5HT: Clostridium bifermentans NCTC1341; $C$. butyricum NCTC7423; $C$. chauvoei NCTC8070; $C$. difficile NCTC11223; C. fallax NCTC8380; C. histolyticum NCTC nos. 503, 7123 and 7124; $C$. novyi type A NCTC538, type C NCTC 9747 and type D NCTC8350; $C$. perfringens type A NCTC8237, type B NCTC3110, type C NCTC3180 and type E NCTC8084; C. septicum NCTC547, C. sordelli NCTC8780; $C$. sporogenes NCTC8594; $C$. tertium NCTC541 and $C$. tetani NCTC249. C. irregularis NCIB11830 (ATCC25756) was obtained from the National Collection of Industrial Bacteria, Torry Research Station, Aberdeen. The strains of $C$. botulinum were from the collection in the Botulinum Laboratory, Unilever Research, Sharnbrook, Bedford: type A, strain nos. 1, 7, 8, 9; type B (proteolytic) strain nos. 25, 26, 27; type B (non-proteolytic) strain nos. 188, 194, 229; type C, strain no. 205; type D, strain no. 154; and type E, strain nos. 34, 118,126,250. Other strains included our own laboratory cultures of $C$. cadaveris MPRL34, MPRL506, 50233; C. irregularis 4403; C. nexile MPRL39; C. novyi type A GR2A, GR3A; $C$. novyi type B GR2B; $C$. paraputrificum CP3; $C$. perenne MPRL95; $C$. sporogenes 24,$33 ; C$. subterminale MPRL303; and C. tertium CT1, CT2. Others were obtained from the following sources: C. absonum $\mathrm{Ha} 9103$ and C. paraperfringens 2227 from Professor S. Nishida, Kanasawa University, Japan and C. perfringens type D, L85 from the late Professor C. L. Oakley, University of Leeds. All strains were fully authenticated by performing the biochemical tests described in the Virginia Polytechnic Institute Anaerobe Laboratory Manual (Holdeman et al., 1977).

Culture of strains and preparation of EDTA extracts. Lyophilised cultures were inoculated into Robertson's Cooked Meat Broth $(10 \mathrm{ml})$ and incubated anaerobically with $\mathrm{CO}_{2} 10 \%$ for 16 $\mathrm{h}$ at $37^{\circ} \mathrm{C}$. These cultures $(0.2 \mathrm{ml}$ volumes $)$ were used to inoculate $100-\mathrm{ml}$ volumes of proteose peptone-yeast extract broth (Holbrook, Duerden and Deacon, 1977) which were incubated anaerobically with $\mathrm{CO}_{2} 10 \%$ for $16 \mathrm{~h}$ at $37^{\circ} \mathrm{C}$. Spore formation, as determined by phase contrast microscopy, was negligible under these conditions. Antigens were prepared from the $100-\mathrm{ml}$ cultures by extraction with $10 \mathrm{~mm}$ EDTA by the method described previously (Poxton and Byrne, 1981a) except that the dialysis step was found to be unnecessary and was omitted.

Preparation of antisera. Antisera were raised in New Zealand White rabbits by intravenous injection of whole, washed, killed bacteria during a 5-week period (Poxton, 1979). The bacteria were killed by ultraviolet irradiation and $10^{9}$ organisms were used for each injection except for the toxigenic strain of C. sordelli, NCTC $8780\left(10^{6}-10^{7}\right.$ organisms, see Poxton and Byrne, 1981a).

Indirect enzyme-linked immunosorbent assay (ELISA). The EDTA extracts were diluted to contain protein $30 \mu \mathrm{g} / \mathrm{ml}$ in antigen buffer and used to coat wells in flat-bottomed microtitre plates. The ELISA technique was performed as described earlier (Poxton, Brown and Collee, 1982).

Reading and presentation of results. In all titrations, a homologous reaction was included and heterologous reactions were compared directly with this. A reaction was considered significant if the titre was within six doubling dilutions $(\mathrm{T} / 32)$ of the titre of the reference reaction obtained between a reference antiserum and the homologous antigen preparation. The results are expressed as a fraction of the homologous reference titre. The size of the symbol in the figure is proportional to the degree of the reaction or cross-reaction, on this basis. A reaction at a titre of less than $1 / 32$ of the homologous titre was considered insignificant. 


\section{RESULTS}

\section{Reference homologous reactions}

Initially, EDTA extracts prepared from 10 species of Clostridium were used as antigens in an indirect ELISA system with their homologous antisera. During the course of the study, these homologous reactions were included as controls whenever a specific antiserum was used and the range of titres (table) reflects the variation found in repeated titrations.

TABLE

Reactions of EDTA antigens with homologous antisera in an indirect ELISA system

\begin{tabular}{|c|c|c|c|c|c|c|c|c|}
\hline \multirow{2}{*}{$\begin{array}{l}\text { Reference antiserum raised } \\
\text { against (species and strain no.) }\end{array}$} & \multirow{2}{*}{$\begin{array}{c}\text { Range of } \\
\text { homologous } \\
\text { titres (T) }\end{array}$} & \multicolumn{6}{|c|}{ Number of strains reacting to a titre of } & \multirow{2}{*}{$\begin{array}{c}\text { Total number } \\
\text { of strains } \\
\text { tested }\end{array}$} \\
\hline & & $\mathrm{T}$ & $\mathrm{T} / 2$ & $\mathrm{~T} / 4 \mathrm{~T} / 8$ & $\mathrm{~T} / 16$ & $\mathrm{~T} / 32$ & $<\mathrm{T} / 32$ & \\
\hline C. cadaveris (MPRL34) & $12800-25600$ & 1 & & & & 1 & 1 & 3 \\
\hline C. chauvoei (NCTC8070) & $12800-25600$ & 1 & 1 & & & & & 2 \\
\hline C. difficile (NCTC11223) & $25600-51200$ & 6 & 25 & 3 & & & & 34 \\
\hline C. histolyticum (NCTC 503) & $6400-51200$ & 2 & 1 & & & & & 3 \\
\hline C. noryi, type A (NCTC538) & $25600-102400$ & 1 & & & 1 & 1 & & 3 \\
\hline $\begin{array}{l}\text { C. perfringens, type A } \\
\text { (NCTC } 8237)\end{array}$ & $12800-51200$ & 2 & 4 & 1 & & & & 7 \\
\hline C. septicum (NCTC547) & $12800-25600$ & 3 & 2 & & & & & 5 \\
\hline C. sordelli (NCTC8780) & $12800-25600$ & 8 & & & & & & 8 \\
\hline C. sporogenes (NCTC8594) & $6400-25600$ & 1 & & 2 & & & & 3 \\
\hline C. tertium (NCTC541) & $1600-3200$ & 1 & 2 & & & & & 3 \\
\hline
\end{tabular}

\section{Tests with representative strains}

Antigens were prepared from 61 other strains of Clostridium belonging to the 10 species listed in the table; they were then used to compare the titres with these antigens and the 10 antisera (now referred to as reference antisera) with those of the homologous reactions. The results indicated that most of the antigens gave titres within one or two doubling dilutions of the homologous reaction (table). Only the antiserum against $C$. cadaveris failed to react significantly with either of the other two strains tested. The reactions of the C.novyi type A strains were not as strong as those of the other species but a reaction was considered significant if the titre was within six doubling dilutions (T/32) of that of the homologous reaction (see Methods).

\section{Studies of cross-reactions}

With the knowledge that nine of the reference antisera reacted strongly with antigens prepared from several strains of the same species, all 10 reference antisera were used to investigate cross-reactions with antigens from at least two strains of 13 other clostridial species, including five different types of $C$. perfringens and $C$. botulinum, and four of $C$. novyi. The results are summarised in the figure. They are expressed as a fraction of the titre obtained with the reference antiserum and the homologous antigen (see Methods). Three areas of major cross-reactions were identified: in the $C$. perfringens group, in the $C$. difficile/sordelli group, and in the $C$. botulinum/novyi/sporogenes complex. 


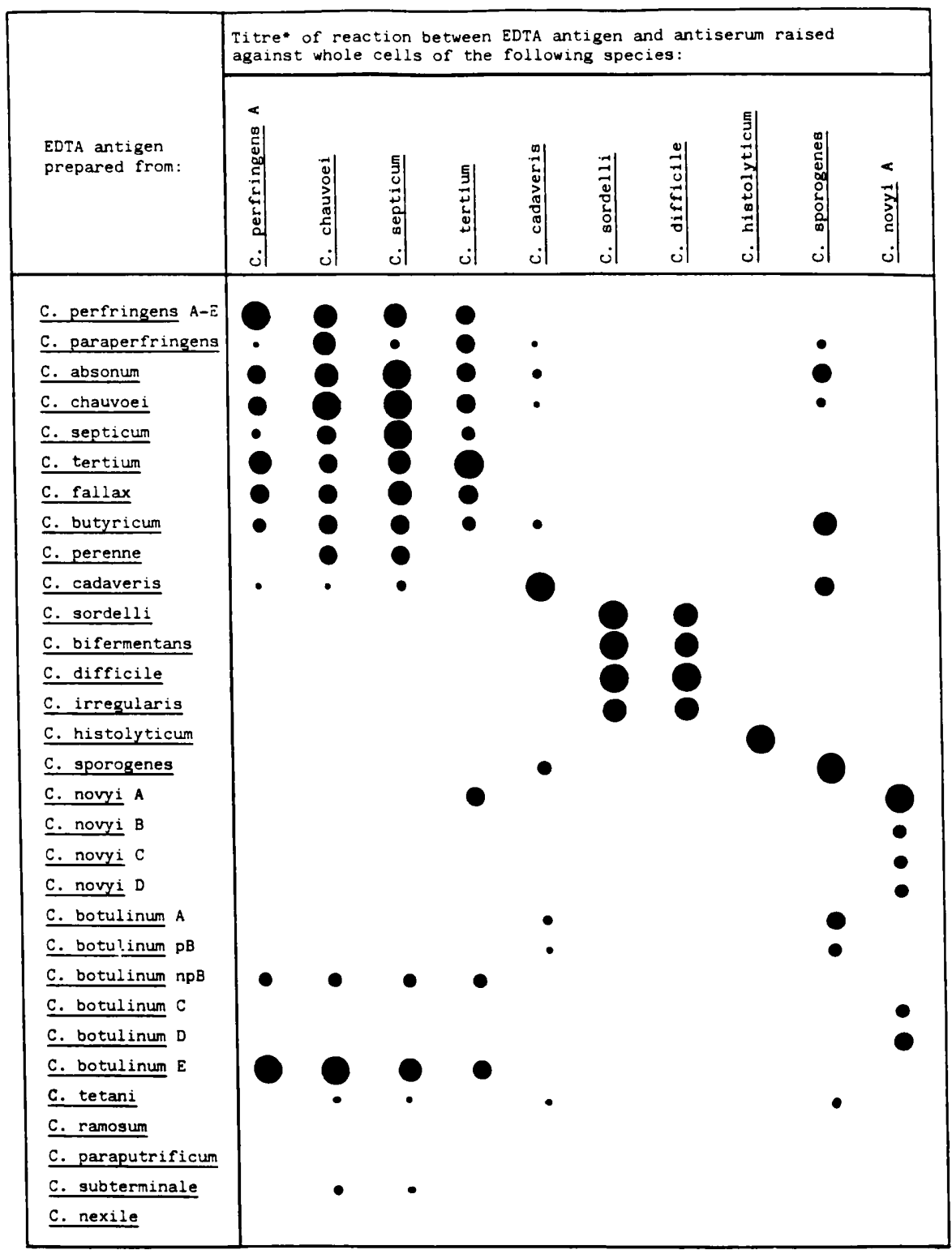

- Titre indicated as a fraction of the homologous titre.

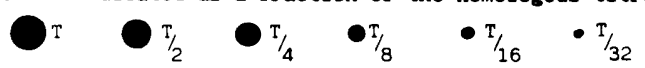

Fig.-Cross-reactions within the genus Clostridium in an indirect ELISA test with EDTA-extracted antigens and whole cell antisera.

\section{DisCUSSION}

The $C$. perfringens group included the first 10 species listed in column 1 of the figure. From previous work, some of the cross-reactions might have been expected, although others were not. All types of $C$. perfringens $(\mathrm{A}-\mathrm{E})$ were indistinguishable by 
this test and they reacted strongly with $C$. chauvoei, $C$. septicum and $C$.tertium antisera as well as with the $C$. perfringens type A reference antiserum. $C$. perfringens, $C$. paraperfringens and $C$. absonum are related biochemically and antigenic relationships could be expected. $C$. chavoei and $C$. septicum are also inter-related but it was unexpected to find that these species reacted strongly with the reference antisera against $C$. perfringens and $C$. tertium. The strong reactions of the above species, together with those of $C$. fallax and $C$. butyricum with the first four reference antisera, and to a lesser extent with the $C$. cadaveris and $C$. sporogenes antisera, confirmed the cross-reactions. Antigens prepared from C. botulinum type B (non-proteolytic) and type $\mathrm{E}$ were also included in this group.

The $C$. sordelli/bifermentans and $C$. difficile group were studied in detail before (Poxton and Byrne, 1981a) but in this extended survey we included the species $C$. irregularis. We have been able to obtain only two strains of $C$. irregularis, one isolated in our own laboratory, strain 4403, and one from the NCIB (strain no. 11830, which is the same as ATCC25756). Both strains were indistinguishable from $C$. difficile, $C$. sordelli and $C$. bifermentans in our ELISA system and shared the same major carbohydrate antigens as these strains (I. R. Poxton, unpublished results). The antigens from these four species did not cross-react with any other antisera, nor did the $C$. sordelli or $C$. difficile antisera cross-react with any other antigen.

It is well established that organisms in the $C$. botulinum/novyi/sporogenes complex possess some biochemical and cultural similarities (Smith, 1977). C. botulinum type A and type B (proteolytic) are biochemically and culturally indistinguishable from $C$. sporogenes; only the toxins separate them. This relationship was confirmed by the ELISA technique which showed the presence of shared antigens. Three of four type-A strains and all of three proteolytic type-B strains reacted with the $C$. sporogenes reference antiserum. Similarly, C. botulinum types $C$ and $D$ are indistinguishable from C. novyi type A (except by toxin), and this was borne out in this study. The unexpected result in this group was the demonstration of cross-reactions between $C$. botulinum type $B$ (non-proteolytic) and type $E$ antigens with the first four reference antisera. Taken in isolation this result may be questioned; however, non-proteolytic type B and type E C. botulinum are distinguished only by toxin production (Smith, 1977) and all of three strains of non-proteolytic type B and all of four strains of $C$. botulinum type $\mathrm{E}$ gave identical results with the four reference antisera, indicating that the result was consistent.

The antisera prepared from $C$. sporogenes and $C$. cadaveris antigens had remarkably similar patterns of specificity. The $C$. cadaveris antiserum, however, may be atypical as antigens prepared from the other two strains did not react significantly with it.

This study has demonstrated some unexpected shared antigens in the genus Clostridium but has confirmed others. The EDTA preparation used as antigen for the C. difficile/sordelli group contained proteins and carbohydrate extracted from the cell surface (Poxton and Byrne, 1981a; Poxton and Cartmill, 1982); the previously unrecognised cross-reactions demonstrated in this study were presumably due to the presence of heat- and formaldehyde-labile antigens not detected in earlier studies. It is difficult to compare our results with those obtained in previous studies which have been used to separate clostridia by immunofluorescent antibody techniques, for such tests to be satisfactory they must be specific, and cross-reacting antibodies were removed by 
absorption. The assay system we have used is relatively non-selective and highly sensitive. We suggest that the results presented here may provide useful guidelines when designing immunological methods for the detection of clostridia and may also help to define taxonomic relationships in the genus. A detailed investigation at the molecular level of some of the more important areas of cross reactions is currently being made.

This study was supported by a grant from the Biomedical Research Committee of the Scottish Home and Health Department (Grant no. K/MRS/50/C247). We gratefully acknowledge the help and advice given by $\mathrm{Dr} \mathrm{J}$. S. Crowther and $\mathrm{Mr} \mathrm{C}$. Grimes of Unilever Research, Bedford, to I.R.P. during culture of the C. botulinum strains at Unilever. We also thank Professor J. G. Collee for his continuing encouragement and advice in the preparation of the manuscript. Part of this study has been included in a thesis submitted by M.D.B. for the award of Fellowship of the Institute of Medical Laboratory Sciences.

\section{REFERENCES}

Batty I, Walker P D 1965 Colonial morphology and fluorescent labelled antibody staining in the identification of species of the genus Clostridium. Journal of Applied Bacteriology 28:112-118.

Cruickshank R, Duguid J P, Marmion B P, Swain R H A Eds. 1975 Clostridium I. In: Medical Microbiology, Vol. 2, 12th ed. Churchill Livingstone, Edinburgh, pp. 471-488.

Hatheway C L, McCroskey L M 1981 Laboratory investigation of human and animal botulism. In: Biomedical aspects of botulism, edited by G. E. Lewis. Academic Press, New York, pp. 165-180.

Holbrook W P, Duerden B I, Deacon A G 1977 The classification of Bacteroides melaninogenicus and related species. Journal of Applied Bacteriology 42:259-273.

Holdeman L V, Cato E P, Moore W E C 1977 Anaerobe laboratory manual, 4th ed. Virginia Polytechnic and State University, Blacksburg, Virginia.

Hubert J, Ionesco H, Sebald M 1981 Détection de Clostridium difficile par isolement sur milieu minimal sélectif et par immunofluorescence. Annals of Microbiology (Institute Pasteur), 132A: 149-157.

Lynt R K, Solomon H N, Kautter D A 1971 Immunofluorescence among strains of Clostridium botulinum and other clostridia by direct and indirect methods. Journal of Food Science $36: 594-599$.

Mandia J W 1955 The position of Clostridium tetani within the serological schema for the proteolytic clostridia. Journal of Infectious Diseases 97:66-72.

Poxton I R 1979 Serological identification of Bacteroides species by an enzyme-linked immunosorbent assay. Journal of Clinical Pathology 32:294-298.

Poxton I R, Brown R and Collee J G 1982 Detection of species-specific and cross-reactive cell-surface antigens of Bacteroides species by an indirect enzyme-linked immunosorbent assay. Journal of Medical Microbiology 15:223-231.

Poxton I R, Byrne M D 1981a Immunological analysis of the EDTA-soluble antigens of Clostridium difficile and related species. Journal of General Microbiology 122:41-46.

Poxton I R, Byrne M D 1981 b Detection of Clostridium difficile toxin by counterimmunoelectrophoresis: a note of caution. Journal of Clinical Microbiology 14:349.

Poxton I R, Cartmill T D I 1982 Immunochemistry of the cell-surface carbohydrate antigens of Clostridium difficile. Journal of General Microbiology 128:1365-1370.

Smith, L DS, 1977 Botulism, the organism, its toxin, the disease. Thomas, Springfield, Illinois pp. 15-33.

Walker P D, Batty I 1964 Fluorescent studies in the genus Clostridium. II. A rapid method for differentiating Clostridium botulinum types $\mathrm{A}, \mathrm{B}$ and $\mathrm{F}$, types $\mathrm{C}$ and $\mathrm{D}$, and type E. Journal of Applied Bacteriology 27: 140-142.

Willis A T 1969 Clostridia of wound infection. Butterworths, London. pp. 1-40. 